\title{
Christian Windler (dir.), Kongressorte der Frühen Neuzeit im europäischen Vergleich. Der Friede von Baden (1714)
}

Niels F. May

\section{(2) OpenEdition}

Édition électronique

URL : http://journals.openedition.org/ifha/9587

DOI : $10.4000 /$ ifha. 9587

ISSN : 2198-8943

\section{Éditeur}

IFRA - Institut franco-allemand (sciences historiques et sociales)

\section{Référence électronique}

Niels F. May, "Christian Windler (dir.), Kongressorte der Frühen Neuzeit im europäischen Vergleich. Der Friede von Baden (1714)», Revue de l'IFHA [En ligne], Date de recension, mis en ligne le 22 septembre 2018, consulté le 24 septembre 2020. URL : http://journals.openedition.org/ifha/9587 ; DOI : https:// doi.org/10.4000/ifha.9587

Ce document a été généré automatiquement le 24 septembre 2020.

(CIFHA 
Christian Windler (dir.), Kongressorte der Frühen Neuzeit im europäischen Vergleich. Der Friede von Baden (1714)

Niels F. May

\section{RÉFÉRENCE}

Christian Windler (dir.), Kongressorte der Frühen Neuzeit im europäischen Vergleich. Der

Friede von Baden (1714), Cologne, Weimar, Vienne : Böhlau, 2016, 303 p., $23 €$ 
Depuis la fin de la guerre de Trente Ans et les traités de Westphalie de 1648, les puissances européennes ont eu recours à de multiples reprises au « congrès ", c'està-dire à une rencontre multilatérale ayant pour but d'éviter ou de clore des guerres d'une portée variable. Ces conflits pouvaient ainsi être délimités à certaines régions ou concerner toute l'Europe (avec parfois même une portée coloniale, comme pour la guerre de Succession d'Espagne). Cette forme de négociation, quasiment inconnue avant le milieu du $\mathrm{XVII}^{\mathrm{e}}$ siècle, devint par la suite une des formes les plus utilisées pour la négociation politique, et ce jusqu'à nos jours. Pour cette raison, ces rencontres ont toujours attiré l'attention des chercheurs en relations internationales.

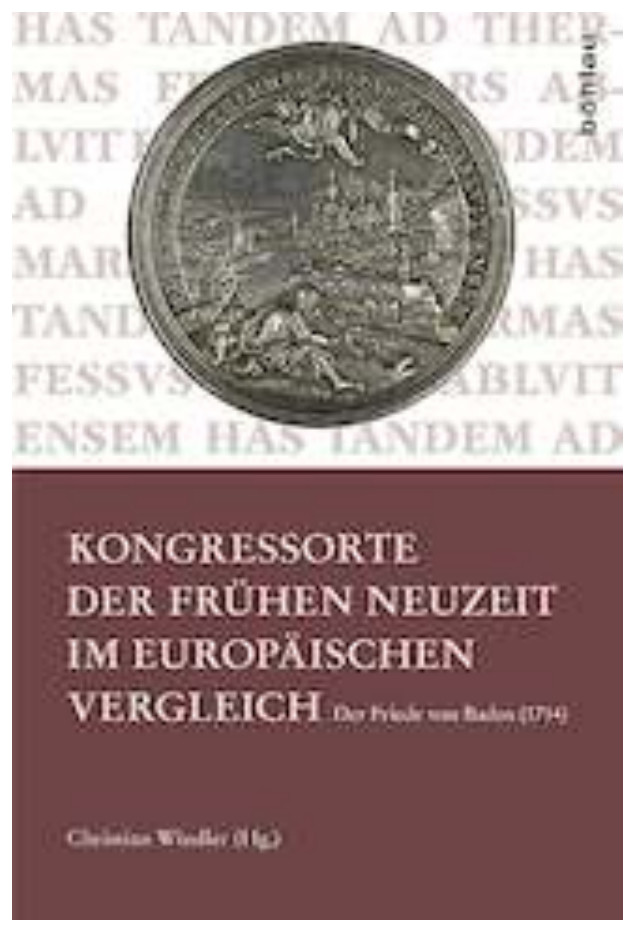
La négociation même et les pourparlers concernant les territoires occupent souvent une place primordiale dans leurs travaux.

Le présent volume, issu d'un colloque commémorant le $300^{\mathrm{e}}$ anniversaire du congrès de Baden en 1714, décale cette perspective pour mettre au centre de l'analyse l'interaction entre la diplomatie et les villes de congrès (14). L'objectif fixé reprend ainsi une proposition d'Heinz Duchhardt visant à marier l'histoire urbaine à l'histoire des relations internationales. Des questions essentielles à l'histoire culturelle du politique aussi bien qu'à l'histoire sociale sont donc au cœur de cette entreprise. Comme le titre l'annonce, le volume ambitionne de comparer les différentes villes de congrès. En tant que directeur du volume, Christian Windler souligne dans son introduction qu'il s'agit d'un champ de recherche jusqu'à présent peu abordé. Seuls Lucien Bély et Gerd Steinwascher ont ouvert des premières pistes dans ce domaine avec leurs travaux sur les traités d'Utrecht et d'Osnabrück (cf. Lucien Bély, Espions et ambassadeurs au temps de Louis XIV, Paris, Fayard, 1990 et Gerd Steinwascher, Osnabrück und der Westfälische Frieden. Die Geschichte der Verhandlungsstadt (1641-1650), Osnabrück, Verein für Geschichte und Landeskunde von Osnabrück, 2000). Pour la plupart des autres congrès, ces questions ne sont pas ou peu abordées.

Les trois parties du présent ouvrage sont précédées d'une première étude dans laquelle Lothar Schilling analyse le processus de sélection et le statut des villes devenues hôtes d'un congrès de paix. Il y montre que les critères de sélection restent semblables entre les traités de Westphalie en 1648 et le traité d'Aix-la-Chapelle en 1748 : les lieux doivent être facilement accessibles pour les participants et dépourvus d'une symbolique forte antérieure aux congrès; l'exercice libre de la religion doit y être garanti pour les différentes confessions et le train de vie des négociateurs doit pouvoir y être assuré. Enfin, point crucial, la sécurité des négociateurs doit être garantie. C'est pourquoi les villes de congrès sont déclarées villes neutres pendant les négociations. L. Schilling 
souligne à juste titre qu'il faut distinguer cette neutralité politique de la neutralité juridique telle qu'elle est discutée dans le droit de gens à l'époque moderne.

La première section du volume analyse ensuite les diètes fédérales réunissant les cantons suisses à Baden et les parallèles possibles avec la diète perpétuelle d'Empire à Ratisbonne. Dans le premier article, Bruno Meier s'intéresse à la culture du Spa à Baden et son importance pour les négociations. Andreas Würgler et Christoph Kampmann mènent ensuite au travers de deux études une comparaison stimulante entre les fonctionnements de la diète fédérale et de la diète perpétuelle. L'étude de C. Kampmann, qui se réfère aux résultats de A. Würgler, montre en particulier combien l'analyse historique gagne par une juxtaposition des différents éléments structurant la diète fédérale et la diète perpétuelle.

La deuxième section est consacrée au congrès de Baden de 1714 en tant que tel congrès qui avait avant tout, comme l'a montré Rudolf Stücheli, la fonction de légitimer le contenu du traité de Rastatt, conclu peu de temps avant. Thomas Lau montre que l'année 1714 ne peut pas être considérée comme celle de la mise en place de la neutralité suisse, à l'encontre de ce qu'avaient souligné Paul Schweizer et Edgar Bonjour dans leurs études classiques. Selon T.Lau, il s'agit d'une neutralité aux conséquences ambiguës - une neutralité qui réunissait et divisait à la fois les cantons suisses et ne représentait donc pas un moment unificateur. C'était avant tout la proximité des cantons suisses avec les zones de combats qui les amenait à être neutres, neutralité émanant donc d'une décision pragmatique. Rolf Stüchli donne ensuite dans son article une vue d'ensemble sur le contexte et les sujets de discussion. La section se clôt par une analyse de Guido Braun portant sur l'envoyé papal Domenico Passionei, déjà présent à Utrecht. Sa mission diffère clairement de celles des nonces au XVII siècle lors des rencontres internationales. Bien qu'exclu des négociations officielles, il interagit en permanence avec les négociateurs - aussi bien catholiques que protestants - lors des rencontres informelles.

La troisième partie est enfin consacrée à la comparaison des villes de congrès à l'époque moderne. Gerd Steinwascher et Anuschka Tischer analysent le rôle de Münster et d'Osnabrück lors des négociations westphaliennes (1643-1648). La contribution de G. Steinwascher renverse la perspective habituelle en envisageant les négociations à partir de la ville. Il peut ainsi montrer que la signature du traité de paix fut pour la ville d'Osnabrück une perte, aussi bien au niveau politique qu'économique. A. Tischer analyse la structure des négociations westphaliennes, ayant lieu en même temps dans deux villes, et les problèmes liés à cette négociation partagée. Elle indique que la division des négociations entre Münster et Osnabrück n'est pas uniquement due à la confession des parties négociantes. Tilman Haug consacre son étude au congrès de Cologne qui échoua en 1675 et au congrès de Nimègue (1676-1678), en se concentrant d'une part sur la neutralité des villes et la sécurité des diplomates et d'autre part sur la fonction des rencontres officieuses lors des négociations. Lucien Bély montre par la suite les changements provoqués dans la vie à Utrecht par le congrès et comment les négociateurs se confrontent parfois aux hostilités des habitants à l'égard des négociateurs «étrangers ". Renger de Bruin et David Onnekink consacrent quant à eux leur étude aux pratiques religieuses à Utrecht. Leur contribution s'inscrit dans un contexte historiographique plus large qui questionne la sécularisation progressive des relations internationales. Hillard von Thiessen résume les résultats du volume en 
développant la définition d'une «diplomatie de congrès » et en soulignant les champs de recherche qui restent à traiter.

Le présent volume donne de multiples aperçus nouveaux sur les différentes villes de congrès et illustre comment la ville et les négociations entrent en interaction lors des congrès. Il faut cependant noter que la comparaison figurant dans le titre du livre doit être faite en grande partie par le lecteur. Les thématiques abordées dans les différentes contributions varient, ce qui est dû à l'absence des recherches préliminaires pour la plupart des questions abordées, comme le souligne le directeur du volume. Certains thèmes, à l'instar de la neutralité ou de l'exercice de la religion, sont récurrents tandis que d'autres questions sont analysées d'une manière plus sporadique, telles que la transformation de la vie urbaine causée par exemple par la prostitution ou le rôle des négociations officieuses. Si les congrès et leurs complexités, qui concernent tant l'objet que les sources, peuvent être un obstacle à une vue d'ensemble que pourrait rechercher un seul chercheur, une grille d'analyse plus précise pour les contributeurs aurait facilité le travail de synthèse du lecteur. Par ailleurs, il convient de relever que les contributeurs sont presque tous des spécialistes de la diplomatie, les spécialistes de l'histoire urbaine étant sous-représentés. Néanmoins, une inspiration mutuelle entre l'histoire des relations internationales et l'histoire urbaine semble une entreprise souhaitable qui pourrait enrichir les deux champs. De ce point de vue, le présent volume ouvre des pistes prometteuses pour des recherches ultérieures.

\section{INDEX}

Index chronologique : Période moderne

Thèmes : Histoire des États et des pouvoirs

\section{AUTEURS}

NIELS F. MAY

Institut historique allemand de Paris 